\title{
MAGNETOSTATIC ENERGY ASSOCIATED WIT́H VARIATION OF LOCAL MAGNETIZATION DIRECTION IN FIELD ANNEALED AMORPHOUS RIBBONS
}

\author{
I. Ciobotaru, N. Rezlescu and R. Anghelache \\ Institute of Technical Physics, D. Mangeron 47, Iasi 6600, Romania
}

\begin{abstract}
It is shown that in field annealed amorphous ribbons (with oblique intrinsic anisotropy), the magnetostatic energy associated with spatial fluctuations of the local magnetization depends on the magnetization state. The corresponding magnetostatic interactions can play an important part in the magnetic behaviour of the amorphous ribbons with small macroscopic anisotropy.
\end{abstract}

PACS numbers: $75.50 . \mathrm{Kj}$

Some amorphous ribbons show distinct magnetic properties due to their small macroscopic anisotropy. In the amorphous samples, the magnetocrystalline anisotropy is absent but the magnetoelastic anisotropy, the unidirectional order or the shape effect can play an important part. Still another characteristic is the fluctuation of the magnetic moment around the average magnetization of the magnetic domain $[1,2] \boldsymbol{M}_{\mathrm{D}}$. Since the divergence of the vector of local magnetization $\boldsymbol{M}$ is not zero, magnetostatic interactions between the neighbouring regions of the sample will be present. These interactions can play an important part in the domain pattern formation in the case of longitudinal anisotropy [3], as well as in the magnetic and magnetoelastic behaviour of the amorphous ribbons in low field [4].

The aim of this paper is to calculate the density of the magnetostatic energy $E_{v}$ associated to the volume poles density in amorphous ribbons with oblique anisotropy. For this, we shall start from the general formula for a rectangular sample with the thickness $2 d$ and the periodic variation of the magnetization in the sample plane [5]

$$
E_{v}=\frac{\mu_{0}}{2} \sum_{k \neq 0} \frac{\left(k \cdot M_{k}\right)\left(k \cdot M_{-k}\right)}{k^{2}}\left[1-\frac{1-\exp (-2 k d)}{2 k d}\right],
$$

where $\mu_{0}$ is the free space permeability, $k$ is the wave vector in the ribbon plane and $\boldsymbol{M}_{k}$ are the Fourier coefficients. The problem consists in adopting a model related to the local magnetization orientation and the calculus of the Fourier coefficients. 
Given the chemical and topological disorder characterising the amorphous structure, the tensor of local anisotropy presents spatial fluctuations with a correlation length given by the local order radius. The direction of the magnetic moment is given by the balance between the exchange force, the local anisotropy and macroscopic anisotropy forces [2]. The average magnetization (domain magnetization) $M_{\mathrm{D}}$ results over which a distribution of the direction of local magnetization $\boldsymbol{M}$ is superposed. In transverse field annealed sample, the technical saturation magnetization $M_{\mathrm{D}}$ (for the magnetic field $H \simeq 10^{2}-10^{3} \mathrm{~A} / \mathrm{m}$ ) is smaller than the magnetization obtained for strong fields $[6]\left(H \simeq 10^{5} \mathrm{~A} / \mathrm{m}\right)$. Thus for the whole region of technical magnetization there are fluctuations of the local magnetization direction. The existence of these fluctuations was revealed by the Bitter technique [1]: beside the walls separating the large domains, a system of auxiliary walls was noticed, transverse to $\boldsymbol{M}_{\mathrm{D}}$ due to the stray field. In order to describe the spatial variation of the local magnetization direction, we shall consider the model presented in [3]: the angle $\varepsilon$ between the local magnetization and the domain magnetization presents a sinusoidal variation along the vector $M_{\mathrm{D}}$ with the amplitude $\gamma$ in the ribbon plane

$$
\varepsilon=\gamma \sin \left(\frac{\pi y^{\prime}}{l}\right)
$$

where $l$ is the distance separating two secondary walls and $x^{\prime} O^{\prime} y^{\prime}$ is the local coordinate system attached to a magnetic domain. $\left(O y^{\prime} \| M_{\mathrm{D}}\right)$. Let us consider a field annealed amorphous ribbon. We choose a system of coordinates with the origin in the ribbon centre so that the $x O y$ plane overlaps on the median plane of the sample and the $O x$ axis is normal to the domain walls (Fig. 1). Under the influence of an external field $\boldsymbol{H}$, the vector $\boldsymbol{M}_{\mathrm{D}}$ rotates making with the $O x$ axis the angle $\theta$ in the II domain and $2 \pi-\theta$ in the domains I and III and the volume fraction where domain magnetization makes the angle $\theta$ will become $v>0.5$. The local coordinate systems are: $x_{\mathrm{I}} O_{\mathrm{I}} y_{\mathrm{I}}, x_{\mathrm{II}} O_{\mathrm{II}} y_{\mathrm{II}}$ and $x_{\mathrm{III}} O_{\mathrm{III}} y_{\mathrm{III}}\left(O_{\mathrm{II}} \equiv O\right)$. The origins $O_{\mathrm{I}}$ and $O_{\mathrm{III}}$ are shifted in relation with $O$ with $(-L, L(2 v-1) \cot \theta)$ and $(L,-L(2 v-1) \cot \theta)$ respectively ( $L$ being the magnetic domain width). The

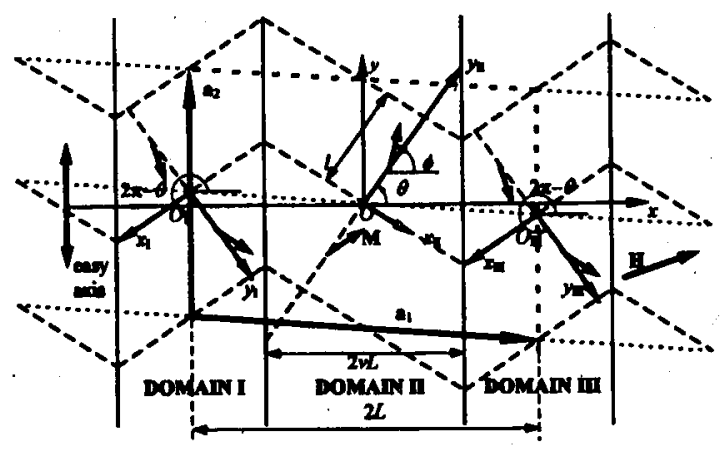

Fig. 1. Domain structure. 
angle $\phi$ between the local magnetization vector and the $O x$ axis is

$$
\phi=\left\{\begin{array}{l}
2 \pi-\theta+\gamma \sin \left(\frac{\pi}{l}(-y \sin \theta+(x+2 v L) \cos \theta)\right) \quad \text { if } x \in(-L,-v L) \\
\theta+\gamma \sin \left(\frac{\pi}{l}(y \sin \theta+x \cos \theta)\right) \quad \text { if } x \in(-v L, v L) \\
2 \pi-\theta+\gamma \sin \left(\frac{\pi}{l}(-y \sin \theta+(x-2 v L) \cos \theta)\right) \quad \text { if } x \in(-L,-v L) .
\end{array}\right.
$$

In order to obtain the Fourier coefficients for the functions $\cos \phi$ and $\sin \phi$ we use the Bessel-Fourier expansion

$$
\mathrm{e}^{\mathrm{i} \phi}=\left\{\begin{array}{l}
\mathrm{e}^{\mathrm{i} \theta} \sum_{m} J_{-m}(\gamma) \mathrm{e}^{-\mathrm{i} m \frac{\pi}{T}(x+2 v L) \cos \theta} \mathrm{e}^{\mathrm{i} m \frac{\pi}{T} y \sin \theta} \quad \text { if } x \in(-L,-v L) ; \\
\mathrm{e}^{\mathrm{i} \theta} \sum_{m} J_{m}(\gamma) \mathrm{e}^{\mathrm{i} m \frac{\pi}{\gamma} x \cos } \mathrm{e}^{\mathrm{i} m \frac{\pi}{T} y \sin \theta} \text { if } x \in(-v L, v L) \\
\mathrm{e}^{\mathrm{i} \theta} \sum_{m} J_{-m}(\gamma) \mathrm{e}^{-\mathrm{i} m \frac{\pi}{T}(x-2 v L) \cos \theta} \mathrm{e}^{\mathrm{i} m \frac{\pi}{\tau} y \sin \theta} \text { if } x \in(v L, L)
\end{array}\right.
$$

with $m$ integer. The translational symmetry is defined by two fundamental vectors

$$
a_{1}=2 L(1,-(2 v-1) \cot \theta) ; \quad a_{2}=\frac{2 l}{\sin \theta}(0,1)
$$

and the wave vector is given by the expression

$$
k_{n, m}=\frac{\pi}{L}((2 v-1) m \rho \cos \theta+n, m \rho \sin \theta)
$$

with $n$ integer and $\rho=L / l$. The Fourier coefficient $M_{n, m}$ are given by

$$
M_{n, m}=\frac{1}{\left|a_{1} \times a_{2}\right|} \quad \iint_{\text {(fundamental cell) }} M(x, y) \mathrm{e}^{-\mathrm{i}\left(k_{n, m}^{x} x+k_{n, m}^{y} y\right)} \mathrm{d} x \mathrm{~d} y .
$$

The average value of $M(x, y)$ has the components

$$
M_{0.0}^{x}=M_{L} J_{0}(\gamma) \cos \theta ; \quad M_{0.0}^{y}=M_{L} J_{0}(\gamma)(2 v-1) \sin \theta,
$$

$M_{L}$ being the local magnetization magnitude. From Eq. (8) it follows that $M_{\mathrm{D}}=$ $M_{L} J_{0}(\gamma)$. Using Eqs. (1) and (5)-(7) with $x 1=((2 v-1) m \rho \cos \theta+n)^{2}+(m \rho \sin \theta)^{2}$, $x 2=(2 v-1) m \rho \cos \theta+n \pm m \rho \cos \theta, x 3=(2 v-1) m \rho \cos \theta+n+m \rho \cos \theta, x 4=$ $(2 v-1) m \rho \cos \theta+n-m \rho \cos \theta$, we can obtain the expression of $E_{v}$

$$
\begin{aligned}
E_{v}= & \frac{2}{\pi^{2}} \mu_{0} M_{L}^{2} \sum_{\substack{n, m(m \text { even }) \\
x 1 \neq 0, \quad x 2 \neq 0}} \frac{J_{m}^{2}(\gamma) m^{2} \rho^{2}(n+m \rho(2 v-1) \cos \theta)^{2}}{((2 v-1) m \rho \cos \theta+n)^{2}+(m \rho \sin \theta)^{2}} \\
& \times \frac{\sin ^{2}(\pi v(n-2 m \rho(1-v) \cos \theta))}{\left(((2 v-1) m \rho \cos \theta+n)^{2}+(m \rho \cos \theta)^{2}\right)^{2}} \\
& \times\left(1-\frac{1-\mathrm{e}^{-2 \pi \frac{d}{L} \sqrt{((2 v-1) m \rho \cos \theta+n)^{2}+(m \rho \sin \theta)^{2}}}}{2 \pi \frac{d}{L} \sqrt{((2 v-1) m \rho \cos \theta+n)^{2}+(m \rho \sin \theta)^{2}}}\right) \\
& +\frac{1}{8} \mu_{0} M_{L}^{2}(1-v)^{2} \sum_{\substack{n, m(m \text { even }) \\
x 1 \neq 0, x 3=0}} J_{m}^{2}(\gamma)\left(1-\frac{1-\mathrm{e}^{-2 \pi \frac{d}{L}|m|}}{2 \pi \frac{d}{L}|m|}\right) \\
& +\frac{1}{8} \mu_{0} M_{L}^{2} v^{2} \sum_{\substack{n, m(m \text { even }) \\
x 1 \neq 0, x 4=0}} J_{m}^{2}(\gamma)\left(1-\frac{1-\mathrm{e}^{-2 \pi \frac{d}{L}|m|}}{2 \pi \frac{d}{L}|m|}\right)
\end{aligned}
$$


The above equation gives the density of the magnetostatic energy $E_{v}$ associated to the volume poles density in amorphous ribbons with oblique anisotropy. Since the Fourier coefficients and the wave vector depend on the orientation of the domain magnetization $\theta$ and the volume fraction $v$, it follows that $E_{v}$ is dependent on the technical magnetization state $(\theta$ and $v)$. In the case of longitudinal anisotropy, $\left(\boldsymbol{H} \| O y ; \theta=\pi / 2\right.$ and the reduced magnetization $\left.m_{r}=2 v-1\right) \mathrm{Eq}$. (9) reduces to Eq. (A5) given in Ref. [3]. In this case the magnetostatic interaction due to the spatial variation of the local magnetization direction can contribute to the occurrence of large domain pattern [3] $\left(10^{-4}-10^{-3} \mathrm{~m}\right)$. In the case of the transverse anisotropy $\left(v=1 / 2 ; \boldsymbol{H} \| O x\right.$ and $\left.m_{r}=\cos \theta\right)$ the domain width is smaller and depends on the ribbon width.

Equation (9) shows that the magnetostatic interaction due to the spatial fluctuation of the local magnetization occurs in amorphous ribbons with oblique anisotropy. Numerical results given by (9) show that the energy density $E_{v}$ increases with the magnetization. The difference of the energy density between the saturation and demagnetized states is $\simeq 25 \mathrm{~J} / \mathrm{m}^{3}$ and the energy density for demagnetized state is $\simeq 100 \mathrm{~J} / \mathrm{m}^{3}$ (for the following values of the parameters: $\mu_{0} M_{L}=1 \mathrm{~T}, l=L=2 d=40 \times 10^{-6} \mathrm{~m}$ and $\left.\gamma=\pi / 10\right)$. Since the intrinsic anisotropy constant of the field annealed amorphous ribbons can be lower than $10^{2} \mathrm{~J} / \mathrm{m}^{3}$ it follows that the magnetostatic interactions can play an important part in the magnetic and magnetoelastic behaviour. The increase $\Delta E_{v}(\theta, v)=$ $E_{v}(\theta, v)-E_{v}(\pi / 2,1 / 2)$ can be considered as a magnetic domain coupling energy of magnetostatic origin.

\section{References}

[1] P.T. Squire, A.T. Thomas, M.R.J. Gibbs, H. Kuźminski, J. Magn. Magn. Mater. 104-107, 109 (1992).

[2] A.P. Thomas, M.R.J. Gibbs, J. Magn. Magn. Mater. 103, 97 (1992).

[3] I. Ciobotaru, J. Magn. Magn. Mater. 146, 137 (1995).

[4] I. Ciobotaru, N. Rezlescu, J. Phys., Condens. Matter C 7, 1689 (1995).

[5] A. Corciovei, Gh. Adam, J. Phys. Collog. 32, C1-408 (1971).

[6] H.T. Savage, M.L. Spano, J. Appl. Phys. 53, 8092 (1982). 